\title{
Leader
}

\section{Biological consequences of the BCR/ABL fusion gene in humans and mice}

\author{
M Y Gordon
}

The BCR/ABL fusion gene is pathognomonic for the human disease chronic myeloid leukaemia (CML). CML is a malignant myeloproliferative disorder that is characterised at the cytogenetic level by the Philadelphia (Ph) translocation between chromosomes 9 and 22 . At the molecular level this results in the juxtaposition of parts of the BCR and ABL genes to form a fusion gene (that is, BCR/ABL). The p2 $10^{\text {bcr/abl }}$ protein tyrosine kinase product of $\mathrm{BCR} / \mathrm{ABL}$ is presumed to be responsible for the biological and clinical features of CML in humans. A vast amount of work has been done on the molecular biology and biochemistry of p2 $10^{\text {bcr/abl }}$ (reviewed in $^{12}$ ) but this article is concerned with the cell biological consequences of p210 ${ }^{\text {bcrabl }}$ expression.

\section{BCR/ABL in humans}

CML is a biphasic or triphasic disease beginning with a chronic phase and then progressing through a variable accelerated phase into an acute phase (blast crisis) resembling an acute myeloid or lymphoid leukaemia. A clinical description of chronic phase CML includes a raised white cell count, predominantly of well differentiated cells of the granulocytic series, and hepatosplenomegaly as a result of myeloid infiltration of the liver and spleen. Since the $\mathrm{Ph}$ chromosome is found in the less expanded erythroid and megakaryocytic series, as well as in the myeloid series, the original lesion must have occurred in a primitive multipotent haemopoietic stem cell. In contrast to normal haemopoiesis, progenitor cells of all lineages (CFU-GM, BFU-E, and so on) circulate in large numbers in the blood of CML patients.

The myeloid expansion in CML must be the result of an imbalance between proliferation (self renewal) and differentiation at some stage in the haemopoietic cell maturation pathway. In normal haemopoiesis, the balance between these two outcomes of stem cell division (assuming that apoptosis does not play a major role) is responsible for maintaining the steady state output of mature blood cells..$^{3-5}$ As CML is a stem cell disease, the imbalance could, theoretically, occur at any stage from the stem cell to the last proliferating cell type (that is, promyelocyte) in the pathway. That expansion in number occurs at the stem cell level is supported by data showing that the primitive progenitor cells (surrogate stem cells) that can be assayed in vitro are increased in CML. ${ }^{6}$ However, the numbers of later myeloid progenitors, the CFUGM, are increased to a greater extent, indicating that expansion of the committed progenitor cells may make a significant contribution to the myeloid mass in CML. ${ }^{5}$

The size of the stem cell compartment is regulated by equal numbers of stem cell progeny remaining in the stem cell pool (proliferation or self renewal) or leaving it by differentiation or apoptosis. Thus an increase in the size of the stem cell population could be caused by a reduction in differentiation or apoptosis (thereby increasing the probability of self renewal), although there is no direct evidence that either is the case. However, CML stem cell surrogates give rise to fewer CFU-GM in vitro than their normal counterparts, ${ }^{6}$ which may reflect reduced differentiation. Primitive progenitor cells in CML do not show a reduced susceptibility to apoptosis in in vitro test systems. $^{7}$

In the more mature progenitor cell compartment, the positive arm is provided by input as well as by self renewal; the negative arm again consists of differentiation plus apoptosis. As indicated above, the primitive cells produce subnormal numbers of CFU-GM so that CFU-GM expansion as a result of raised input from a pre-CFU-GM progenitor seems unlikely. ${ }^{6}$ A reduced level of cell loss from the CFU-GM compartment by apoptosis is controversial. Some studies indicate that CML CFU-GM are less susceptible than normal CFU-GM to apoptosis ${ }^{8}{ }^{9}$; others do not. ${ }^{70-13}$ We have studied the proliferation of CFU-GM in normal and CML blood and in normal bone marrow by growing CFU-GM colonies, replating them into secondary cultures, and observing secondary colony formation. ${ }^{14}$ This procedure provides a measure of the numbers of CFU-GM produced during colony formation and hence a measure of self renewal at the CFU-GM level. In CML this value is greater than normal. Interestingly, the ability of CML CFU-GM to self renew is suppressed by interferon $\alpha$, which has been used successfully in the treatment of CML, but self renewal by normal CFU-GM is not suppressed. ${ }^{14}$ 
The above discussion relates to the outcome of cell division rather than the numbers of cell divisions per unit time (that is, cell cycle duration or proliferation rate). Some studies have suggested that the proliferation rate in CML is increased $^{15}$; others have not. ${ }^{16}{ }^{17}$ An increased proliferation rate of CML progenitor cells could amplify their growth advantage over normal cells if their self renewal capacity is increased.

An increased proliferation rate could reflect insensitivity to negative regulators of haemopoietic progenitor cell proliferation. Normal haemopoiesis is found in the microenvironment of the bone marrow, where stromal cells are candidate negative regulators of haemopoiesis. ${ }^{18}$ Primitive CML cells are able to escape from the marrow microenvironment, because their ability to bind to stromal cells is defective, ${ }^{1920}$ and hence avoid its negative restraining influence. Another negative regulator to which CML progenitor cells may be subnormally sensitive is the chemokine macrophage inflammatory protein $1 \alpha(\mathrm{MIP}-1 \alpha) .^{21} 22$

An important feature of CML is that $\mathrm{Ph}$ negative, presumably normal, progenitor cells coexist with the $\mathrm{Ph}$ positive leukaemic progenitor cells, although the relative numbers of $\mathrm{Ph}$ positive and $\mathrm{Ph}$ negative cells are unclear and may vary in different disease stages. The fact that $\mathrm{Ph}$ negative cells appear to be relatively more frequent in the more primitive progenitor cell compartments ${ }^{23}{ }^{24}$ suggests that normal cell maturation may be inhibited in the presence of CML cells. However, in circumstances where self renewal by normal cells might be expected to increase, the normal cells may in fact have a proliferative advantage over the CML cells. This view is supported by observations of $\mathrm{Ph}$ negative haemopoiesis following autografting ${ }^{25}$ or treatment with interferon $\alpha,{ }^{26}$ and has given rise to the so called tortoise- $v$-hare hypothesis, ${ }^{15}$ which proposes that the self renewal capacity of regenerating normal stem cells is greater than that which can be achieved by CML stem cells. Also, in the long term bone marrow culture system, where conditions are likely to favour normal cell proliferation over differentiation, CML cells show a distinct disadvantage compared with normal cells. ${ }^{15}$ However, in steady state conditions, the self renewal capacity of the CML stem cells is greater than that of normal stem cells.

In addition to the in vitro analysis of clonogenic cells from CML patients, various strategies have been used in attempts to produce an in vivo model of CML in mice. First, murine cells have been manipulated to express $\mathrm{p} 210^{\mathrm{bcr} / a b l}$, either by retrovirus mediated gene transfer or the construction of transgenic animals; second, primary cells from CML patients have been xenografted into severely immunocompromised murine recipients.

\section{BCR/ABL in normal mice}

Several studies have investigated the transplantation of BCR/ABL transduced murine haemopoietic cells into syngeneic mice and found that it causes a CML-like myeloproliferative syndrome in some recipients. However, other recipients may develop a variety of proliferative disorders such as myelomonocytic leukaemia or B cell lymphomas. ${ }^{27-32}$ Another approach taken by Clarke et $a l^{33}$ was to inject p210 $10^{\text {bcrabl }}$ expressing retrovirus intrathymically, which resulted in the development of thymomas after a relatively long latent period.

The latency of the myeloproliferative syndrome has varied from three to five weeks to more than 20 weeks. ${ }^{27-32}$ Gishizky et al discriminated between groups of animals with early ( $<20$ weeks) and late ( $>20$ weeks) onset disease. ${ }^{29}$ Blood counts were raised with differential increases in mature and immature cells of the granulocytic lineage. The mice have a hypercellular marrow and hepatosplenomegaly with extramedullary haemopoiesis in liver and spleen. In general, molecular analyses of the myeloproliferative disease suggests that it arises from one or a few transduced stem cells. This may be the result of clonal selection in vivo or reflect the fact that engraftment of the transduced cells is an inefficient process.

The capacity for serial passage of the myeloproliferative disorder is important because the disease in the primary animal may be aggressive and fatal $^{30}$ and because transformation to acute phase may not occur in the first recipient. Moreover, serial transplantation allows cells from one myeloproliferative disease clone to be transferred to several recipients. Some secondary recipients develop the chronic myeloproliferative syndrome while others develop acute leukaemias of lymphoid or myeloid phenotype. Analysis of the acute leukaemias in the secondary animals confirmed that they were derived from the clones that gave rise to CML in the primary animal. In some cases, the BCR/ABL positive clone produced myeloid and lymphoid disease in separate secondary recipients which shows that the original disease was initiated by a lymphomyeloid stem cell. Gishizky et al compared the serial transplantability of myeloproliferative disease from mice with early and late onset disease. ${ }^{29}$ The cells from mice with late onset disease transplanted more efficiently than those from mice with early onset disease, and gave rise mainly to acute leukaemias in the secondary recipients, with an occasional reiteration of the chronic disorder

\section{BCR/ABL in transgenic mice}

The construction of transgenic mice offers an alternative approach to investigation of the biological consequences of the BCR/ABL chimaeric gene product in vivo. Hariharan et al generated $\mathrm{p} 210^{\mathrm{bcr} / \mathrm{vabl}}$ transgenic mice and reported that some of them developed $\mathrm{B}$ or $\mathrm{T}$ cell lymphomas after variable latent periods. ${ }^{34}$ Subsequently, Honda et al generated transgenic $\mathrm{C} 57 \mathrm{Bl} 6 \times \mathrm{DBA} / 2$ mice expressing p $210^{\text {bcrabl }}$ driven by the metalloproteinase enhancer/promotor. ${ }^{35}$ Two of six founder mice and the transgenic progeny developed leukaemia when they were several months old. The leukaemias were committed to the $\mathrm{T}$ cell lineage and were monoclonal, showing rearrangements of the $\mathrm{T}$ cell receptor loci. Moreover, the p2 $10^{\text {bcrabl }}$ transgene product had enhanced 
kinase activity and increased tyrosine phosphorylation of cellular proteins was observed. No tumours of non-haemopoietic tissue were found, suggesting that the oncogenic activity is limited to the haemopoietic system. However, there was no evidence of granulocytic hyperplasia. Consequently, the $\mathrm{T}$ cell leukaemias were considered to be de novo diseases rather than the result of further events in a "chronic phase" clone. Voncken et al used a different mouse strain $(\mathrm{C} 57 \mathrm{Bl} \times \mathrm{CBA})$ and found $\mathrm{T}$ and $B$ cell leukaemias and infrequently (one of 21) myeloid leukaemias. ${ }^{36}$ All of the leukaemias were acute and there was no evidence of a chronic phase.

Overall, murine haemopoietic cells modified to express p $210^{\text {bcr/abl }}$ reproduce some of the features of human CML, although the course may be more aggressive than the human disease and in some studies there is a marked propensity for lymphoid transformation.

\section{BCR/ABL in severely}

\section{immunocompromised mice}

Since in vitro culture studies and expression of p $210^{\text {bcrabl }}$ in murine haemopoietic cells have failed to provide an adequate solution to the puzzle concerning the kinetic basis of myeloid expansion in CML, investigators have explored the transplantation of human CML cells into immunocompromised murine hosts. It has been suggested that this approach may provide an improved model of the human disease because regulatory sequences upstream from or within the BCR gene, which may be missing from transduced or transgenic mouse cells, can be assumed to be present in the transplanted human CML progenitor cells. The various types of inbred immunocompromised mice that have been developed as hosts for human haemopoietic tissue have been reviewed by Uckun $^{37}$ and Dick. ${ }^{38}$ Both severe combined immunodeficient (SCID) and non-obese diabetic (NOD)/SCID mice have been used as hosts in the study of human CML cells.

The successful engraftment of CML in SCID or NOD/SCID mice depends on the injection of relatively large numbers of cells. For example, Dazzi and colleagues ${ }^{39}$ injected $5 \times 10^{6}$ CD34 positive cells (that is, purified progenitor cells), which is equivalent to $\sim 5 \times 10^{8}$ whole bone marrow or blood cells and a dose per kg mouse body weight of $2 \times 10^{10}$ bone marrow cells. Moreover, it is large in relation to the total bone marrow cellularity of a mouse, which has been estimated to be of the order of $5 \times 10^{8}{ }^{40}$ For these reasons, Dazzi et al investigated the destiny of ${ }^{51} \mathrm{Cr}$ labelled CML cells, injected intravenously into NOD/SCID mice, and found that they were rapidly cleared from the bloodstream then sequestered mainly in the lungs and the liver. ${ }^{39}$ Only a few $(1-2 \%)$ of the injected cells lodged in the bone marrow. However, 18 weeks later a median of $21 \%$ (range $5-45 \%$ ) of the cells in the mouse marrow were of human origin. This study showed for the first time that CML cells can increase in number in the marrow of the NOD/SCID mouse and that their growth rate can be documented. It is noteworthy, however, that negligible numbers of human cells could be found in the bloodstream.

Similar results of xenografting CML cells had been reported by Sawyers et al and Sirard et al in SCID mice, ${ }^{41}{ }^{42}$ and by Wang et al and Lewis et al in NOD/SCID mice. ${ }^{43} 44$ Sirard et al injected chronic phase CML cells into SCID mice and found $10-15 \%$ human cells in the bone marrow 30-60 days after transplant ${ }^{42}$; Wang et $a l^{43}$ using NOD/SCID recipients, found higher levels of engraftment $(40-80 \%$ in $25 \%$ of the transplanted animals). As might be expected, cells from CML patients with accelerated or blast phase disease engraft more rapidly and to a higher level than cells from patients with chronic phase. $.^{39} 41-43$

Sirard et al used flow cytometry to discriminate between lymphoid and myeloid engrafted cells. ${ }^{42}$ They found that the majority $(>76 \%)$ of the human (CD45+) cells were CD13+, and $95 \%$ of the human lymphoid cells were CD19+. Dazzi et al were unable to identify CD34+ cells, ${ }^{39}$ but Sirard et al found CD34+ cells in about $50 \%$ of the mice, ranging in number from $1.6 \times 10^{3}$ to $1.5 \times 10^{6}$ in four long bones (two femurs and two tibias). ${ }^{42}$ Similarly, Wang et al found that $2.5 \%$ of the human cells were CD34+ and $40 \%$ of these were Thy- $1+.{ }^{43}$ When cells from engrafted mice were cultured in vitro under conditions permitting the growth of human colony forming cells, colonies of granulocytic, erythroid, macrophage, megakaryocytic, and mixed lineage cells were found.

As normal cells coexist with leukaemic cells in CML patients, Sirard et al used reverse transcriptase polymerase chain reaction (RT-PCR) to analyse the nature of the in vitro colonies grown from human cells in engrafted mouse marrow, and found that $30 \%$ of them were BCR/ABL negative. ${ }^{42}$ This finding was confirmed by cytogenetic analysis and in the studies of Wang et al and Lewis et $a l .^{43}{ }^{44} \mathrm{~A}$ somewhat lower frequency of $\mathrm{Ph}$ negative cells was reported by Dazzi et al. ${ }^{39}$ They used fluorescence in situ hybridisation of probes for BCR and ABL and detected a maximum of $30 \% \mathrm{Ph}$ negative cells in $24 \%$ of engrafted patients' samples. Overall, these studies show that normal cells can engraft immune deficient mice at the same time as $\mathrm{Ph}$ positive CML and that the normal cells, which are in the minority at the beginning of the experiment, might sometimes have a growth advantage over the CML cells (that is, fulfil the tortoise-v-hare hypothesis of Eaves et $a l^{15}$ ). Recently, this hypothesis has been tested by Dazzi and colleagues by comparing the growth rates of human cells in mice transplanted with normal marrow CD34+ or chronic phase CML CD $34+$ cells. ${ }^{45}$ They found that the growth rate of the normal cell population was initially faster than that of the CML population. However, the growth rate of the normal cell population reached a plateau while the CML cells continued to grow at a constant rate.

Thus far, the use of SCID or NOD/SCID mice as hosts for CML cells has shown that it is possible for CML cells to expand in number in the murine in vivo microenvironment and that 
the kinetics of expansion are distinctly different when cells are obtained from chronic phase, accelerated phase, or blast crisis patients. Moreover, the different kinetics of normal and CML cells in this system seem to provide the first proof of the tortoise- $v$-hare hypothesis. The stage is now set to test strategies aimed at maintaining the normal growth advantage or reducing CML cell proliferation in this model, or both.

1 Gordon MY, Goldman JM. Cellular and molecular mechaBr F Haematol 1996;95:10-20.

2 Verfaillie CM. Chronic myelogenous leukemia: from pathogenesis to therapy. 7 Hematother 1999;8:3-13.

3 Gordon MY, Blackett NM. Some factors determining the minimum number of cells required for successful clinical minimum number of cells required for successful clinica

4 Gordon MY, Blackett NM. Reconstruction of the hematopoietic system after stem cell transplantation. Cell Transplantation 1998;7:339-44.

5 Gordon MY, Dazzi F, Marley SB, et al. Cell biology of CML cells. Leukemia 1999;13(suppl 1):S65-71.

6 Marley SB, Lewis JL, Scott MA, et al. Evaluation of "discordant maturation" in chronic myeloid leukaemia using cultures of primitive progenitor cells and their production of clonogenic progeny (CFU-GM). $\mathrm{Br} f$ Haematol 1996;95:299-305.

7 Amos TAS, Lewis JL, Grand FH, et al. Apoptosis in chronic myeloid leukaemia: normal responses by progenitor cells to growth factor deprivation, X-irradiation and glucocorticoids. Br f Haematol 1995;91:387-93.

8 Bedi A, Zenbauer BA, Barber JP, Sharkis SJ, Jones RJ. Inhibition of apoptosis by BCR-ABL in chronic myeloid leukebition of apoptosis by BCR-A
mia. Blood 1994;83:2038-44

9 Bedi A, Barber JP, Bedi GC, et al. BCR-ABL-mediated inhibition of apoptosis with delay of $\mathrm{G} 2 / \mathrm{M}$ transition after DNA damage: a mechanism of resistance to multiple cancer agents. Blood 1995;86:1148-58.

10 Roger R, Isaad C, Pallardy M, et al. BCR-ABL does not prevent apoptotic death induced by human natural killer or prevent apoptotic death induced by human natural killer or

11 Santucci MA, Anklesaria P, Laneuville P, et al. Expression of p210 BCR-ABL increases haematopoietic progenitor cell radiosensitivity. Int F Radiat Oncol Biol Phys 1993;26:881-9.

12 Albrecht T, Schwab R, Henkes M, et al. Primary proliferating immature myeloid cells from CML patients are not resistant to induction of apoptosis by DNA damage and growth factor withdrawal. Br F Haematol 1996;94:501-7.

13 Carlo-Stella C, Dotti G, Mangoni L, et al. Selection of myeloid progenitors lacking BCR/ABL mRNA in chronic myelogenous leukemia patients after in vitro treatment with the tyrosine kinase inhibitor, genestein. Blood 1996;88: 3091-100

14 Gordon MY, Marley SB, Lewis JL, et al. Treatment with interferon- $\alpha$ preferentially reduces the capacity for amplifiinterferon- $\alpha$ preferentially reduces the capacity for amplififrom patients with chronic myeloid leukemia, but spares from patients with chronic myeloid leukemia, but

15 Eaves CJ, Eaves AC. Stem cell kinetics. Bailliere's Clin Haematol 1997;10:233-57.

16 Clarkson BD, Strife A, Wisniewski D, et al. New understanding of the pathogenesis of CML: a prototype of early neoplasia. Leukemia 1997;11:1404-28.

17 Thiele J, Zirbes TK, Lorenzen J, et al. Apoptosis and proliferation (PCNA labelling) in CML: a comparative immunohistological study of bone marrow biopsies following interferon and busulphan therapy. F Pathol 1997;181:316-22.

18 Gordon MY, Lewis JL, Marley SB, et al. Stromal cells negatively regulate primitive haemopoietic progenitor cell

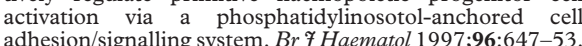

19 Gordon MY, Dowding CR, Riley GP, et al. Altered adhesive interactions with marrow stroma of haematopoietic prointeractions with marrow stroma of haematopoietic pro328:342-4.

20 Verfaillie CM, McCarthy JB, McGlave P. Mechanisms underlying abnormal trafficking of malignant progenitors in chronic myelogenous leukemia: decreased adhesion to stroma and fibronectin but increased adhesion to the basement membrane components laminin and collagen type IV 7 Clin Invest 1992;90:1232.

21 Eaves CJ, Cashman JD, Wolpe SD, et al. Unresponsiveness of primitive chronic myeloid leukemia cells to macrophage inflammatory protein 1 alpha, an inhibitor of primitive normal hematopoietic cells. Proc Natl Acad Sci USA 1993 90:12015.
22 Nirsimloo N, Gordon MY. Progenitor cells in the blood and marrow of patients with chronic phase chronic myeloid

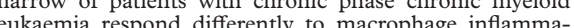
tory factor-1 $\alpha$. Leuk Res 1995;19:319-23.

23 Grand FH, Marley SB, Chase A, et al. BCR/ABL-negative progenitors are enriched in the adherent fraction of CD $34+$ cells circulating in the blood of chronic phase chronic myeloid leukemia patients. Leukemia 1997;11: 1486-92.

24 Verfaillie CM, Bhatia R, Miller W, et al. BCR/ABL-negative primitive progenitors suitable for transplantation can be selected from the marrow of most early chronic phase, but not accelerated phase chronic myelogenous leukemia patients. Blood 1996;87:4770-9.

25 Brito-Bapapulle F, Bowcock SJ, Marcus RE, et al. Autografting for patients with chronic myeloid leukaemia in chronic phase:peripheral blood stem cells may have a finite capacity for maintaining haemopoiesis. Br 7 Haematol 1989;73:76-81.

26 Talpaz M, Kantarjian HM, Kurzrock R, et al. Interferon alpha produces sustained cytogenetic responses in chronic myeloid leukemia. Ann Intern Med 1991;114

27 Daley GQ, van Etten RA, Baltimore D. Induction of chronic myelogenous leukemia in mice by the p210 bcrabl gene of the Philadelphia chromosome. Science 1990;247:824-9.

28 Daley GQ, van Etten RA, Baltimore D. Blast crisis in a murine model of chronic myelogenous leukemia. Proc Natl Acad Sci USA 1991;88:11335-8.

29 Gishizky ML, Johnson-White J, Witte ON. Efficient transplantation of BCR-ABL-induced chronic myelogenous leukemia-like syndrome in mice. Proc Natl Acad Sci USA 1993;90:3755-9.

30 Pear WS, Miller JP, Xu L, et al. Efficient and rapid induction of a chronic myelogenous leukemia-like myeloproliferative disease in mice receiving p210 bcr/abl-transduced bone marrow. Blood 1998;92:3780-92.

31 Kelliher MA, McLaughlin J, Witte ON, et al. Induction of a chronic myelogenous leukemia-like syndrome in mice with v-abl and BCR/ABL. Proc Natl Acad Sci USA 1990;87: 6649-53

32 Zhang X, Ren R. Bcr-Abl efficiently induces a myeloproliferative disease and production of excess interleukin-3 and granulocyte-macrophage colony-stimulating factor in mice: a novel model for chronic myelogenous leukemia. Blood 1998;92:3829-40.

33 Clarke SS, Chen E, Fizzotti M, et al. BCR-ABL and v-abl oncogenes induce distinct patterns of thymic lymphoma involving different lymphocyte subsets. $\mathcal{F}$ Virol 1993;67: 6033.

34 Hariharan IK, Harris AW, Crawford M, et al. A bcr-v-abl oncogene induces lymphomas in transgenic mice. Mol Cell Biol 1989;9:2798.

35 Honda $\mathrm{H}$, Fujii T, Takatoku H, et al. Expression of $\mathrm{p} 210^{\text {bcrabl }}$ by metallothionene promoter induced T-cell leukemia in transgenic mice. Blood 1995;85:2853-61.

36 Voncken JW, Kaarinen V, Pattengale PK, et al. BCR/ABL p210 and p190 cause distinct leukemia in transgenic mice. Blood 1995;86:4603-11.

37 Uckun FM. Severe combined immunodeficient mouse models of human leukemia. Blood 1996;88:1135.

38 Dick JE. Normal and leukemic stem cells assayed in SCID mice. Semin Immunol 1996;8:197-206.

39 Dazzi F, Capelli D, Hasserjian R, et al. The kinetics and extent of engraftment of chronic myelogenous leukemia cells in non-obese diabetic/severe combined immunodeficiency mice reflect the phase of the donorÖs disease: an in vivo model of chronic myelogenous leukemia biology. Blood 1998;92:1390-6.

40 Coggle JE, Gordon MY. Quantitative measurements on the haemopoietic systems of three strains of mice. Exp Hematol 1975;3:181-6.

41 Sawyers CL, Gishizky ML, Quan S, et al. Propagation of human blastic leukemias in the SCID mouse. Blood 1992; 79:2089-98.

42 Sirard C, Lapidot T, Vormoor J, et al. Normal and leukemic SCID-repopulating cells (SRC) coexist in the bone marrow and peripheral blood from CML patients in chronic phase whereas leukemic SRC are detected in blast crisis. Blood 1996;87:1539-48.

43 Wang JCY, Lapidot T, Cashman JD, et al. High level engraftment of NOD/SCID mice by primitive normal and leukemic hematopoietic cells from patients with chronic myeloid leukemia in chronic phase. Blood 1998;91:240614 .

44 Lewis ID, McDiarmid LA, Samels LM, et al. Establishment of a reproducible model of chronic-phase chronic myeloid eukemia in NOD/SCID mice using blood-derived mononuclear or CD34+ cells. Blood 1998;91:630-40.

5 Dazzi F, Boecklin F, Hasserjian R, et al. Normal and chronic phase CML hematopoietic cells repopulate NOD/SCID bone marrow with different kinetics and differentiation pattern [abstract]. Bone Marrow Transplant 1999;23(suppl 1):S58 